\title{
ON THE $p$-RANK OF AN ABELIAN VARIETY AND ITS ENDOMORPHISM ALGEBRA
}

\author{
Josep GonzÁLEZ
}

\begin{abstract}
Let $A$ be an abelian variety defined over a finite field. In this paper, we discuss the relationship between the $p$-rank of $A, r(A)$, and its endomorphism algebra, $\operatorname{End}^{0}(A)$. As is well known, $\operatorname{End}^{0}(A)$ determines $r(A)$ when $A$ is an elliptic curve. We show that, under some conditions, the value of $r(A)$ and the structure of $\operatorname{End}^{0}(A)$ are related. For example, if the center of $\operatorname{End}^{0}(A)$ is an abelian extension of $\mathbb{Q}$, then $A$ is ordinary if and only if $\operatorname{End}^{0}(A)$ is a commutative field. Nevertheless, we give an example in dimension 3 which shows that the algebra $\operatorname{End}^{0}(A)$ does not determine the value $r(A)$.
\end{abstract}

\section{Introduction}

Let $k$ be an algebraically closed field of characteristic $p>0$. Given an abelian variety $A / k$ of dimension $g$, the $p$-rank of $A$ is defined by

$$
r(A):=\operatorname{dim}_{\mathbb{F}_{p}} \operatorname{Pic}^{0}(A)[p]=\operatorname{dim}_{\mathbb{F}_{p}} H^{1}(A, \mathcal{O})^{\mathbb{F}^{*}},
$$

where $F^{*}$ denotes the absolute Frobenius. The value $r(A)$ is invariant under isogenies and satisfies $r(A \times B)=r(A)+r(B)$, for $A, B$ abelian varieties over $k$. Thus, if $A$ is isogenous to a product of abelian varieties $\prod_{i=1}^{m} A_{i}^{n_{i}}$, then $r(A)=\sum_{i=1}^{m} n_{i} r\left(A_{i}\right)$.

Let $\mathcal{C} / k$ be a non-singular projective curve of genus $g>0$. Serre [Se 58] characterized the Hasse-Witt invariant, $r(\mathcal{C})$, by means of the action of $F^{*}$ on the first cohomology group:

$$
r(\mathcal{C})=\operatorname{dim}_{\mathbb{F}_{p}} H^{1}(\mathcal{C}, \mathcal{O})^{F^{*}} .
$$

This research has been partially supported by DGICYT, PB-93-0034. 
If $J$ denotes the jacobian of $\mathcal{C}$, it is clear that $r(J)=r(\mathcal{C})$. Ordinary abelian varieties are those for which $r(A)=g$. Supersingular elliptic curves are those for which $r(A)=0$.

Let us denote by $\operatorname{End}^{0}(A):=\mathbb{Q} \otimes_{\mathbb{Z}} \operatorname{End}(A)$ the endomorphism algebra of $A$. If $E / k$ is an elliptic curve, $E$ is ordinary if and only if $\operatorname{End}^{0}(E)$ is a commutative field (it is equal to $\mathbb{Q}$ or to an imaginary quadratic extension of $\mathbb{Q}) ; E$ is supersingular if and only if $\operatorname{End}^{0}(E)$ is a quaternion algebra over $\mathbb{Q}$. If, in addition, the field $k$ is the algebraic closure of a finite field, the case $\operatorname{End}^{0}(E)=\mathbb{Q}$ is excluded.

The asymptotic behaviour of the Hasse-Witt invariants for the fibres of modular curves, resp. of Fermat curves, has been studied in [Ba-Go 97], resp. [Go 97]. Both cases are quite diferent. It turns out that, for some projective curves over $\mathbb{Q}$, the distribution of the extreme values of the Hasse-Witt invariant of the fibres seems to depend on the type of the endomorphism algebra over $\overline{\mathbb{Q}}$ of their jacobian variety.

In this paper, we summarize some results which show the relationship between $r(A)$ and $\operatorname{End}^{0}(A)$ when the abelian variety $A$ is defined over a finite field. Nevertheless, we provide with an example of two abelian varieties which have $\mathbb{Q}$-isomorphic endomorphism algebras but show different $p$-ranks. One of them is the jacobian of the modular curve $X_{0}(41) / \mathbb{F}_{3}$.

Some of these results are contained in my $\mathrm{PhD}$ thesis. I would like to finish this introduction by expressing my gratitude to my dissertation advisor Prof. Pilar Bàyer for her help and encouragement throughout the realization of the work.

\section{Some general facts}

We fix a positive integer $n$ and consider a power $q=p^{n}$ of the characteristic of $k$. Throughout, $A$ denotes an abelian variety of dimension $g>0$ defined over the finite field $\mathbb{F}_{q}$, and $k=\overline{\mathbb{F}}_{q}$. We denote by $\operatorname{End}_{\mathbb{F}_{q}}(A), \operatorname{resp} . \operatorname{End}(A)$, the ring of endomorphisms of $A$ which are defined over $\mathbb{F}_{q}$, resp. $k$. We write $\operatorname{End}_{\mathbb{F}_{q}}^{0}(A):=\mathbb{Q} \otimes_{\mathbb{Z}} \operatorname{End}_{\mathbb{F}_{q}}(A)$, $\operatorname{End}^{0}(A):=\mathbb{Q} \otimes_{\mathbb{Z}} \operatorname{End}(A)$. If $A$ is $\mathbb{F}_{q}$-isogenous to $\prod A_{i}^{n_{i}}$, where the abelian varieties $A_{i}$ are $\mathbb{F}_{q}$-simple and not $\mathbb{F}_{q}$-isogenous to each other, then $\operatorname{End}_{\mathbb{F}_{q}}^{0}(A)=\oplus M_{n_{i}}\left(\operatorname{End}_{\mathbb{F}_{q}}^{0}\left(A_{i}\right)\right)$, where $M_{n_{i}}$ denotes the ring of $\left(n_{i} \times n_{i}\right)$-matrices.

Let $\varphi \in \operatorname{End}_{\mathbb{F}_{q}}(A)$ be the relative Frobenius endomorphism, whose action on the variety raises to the $q$-th power the coordinates of the points of $A$. For a given prime number $\ell \neq p$, we denote by $T_{\ell}(A)$ the Tate module of $A$, and by $V_{\ell}(A):=\mathbb{Q}_{\ell} \otimes_{\mathbb{Z}_{\ell}} T_{\ell}(A)$. Two abelian varieties $A, B$ defined 
over $\mathbb{F}_{q}$ are $\mathbb{F}_{q}$-isogenous if and only if the corresponding Frobenius have the same characteristic polynomial in the $\ell$-adic representation.

The $\mathbb{Q}$-algebra $\operatorname{End}_{\mathbb{F}_{q}}^{0}(A)$ has $\mathbb{Q}(\varphi)$ as its center. We have that $\operatorname{End}_{\mathbb{F}_{q}}^{0}(A)=\mathbb{Q}(\varphi)$ if and only if the characteristic polynomial of $\varphi$ acting on the Tate module has no double roots. We have that $\mathbb{Q}(\varphi)=\mathbb{Q}$ if and only if $A$ is $\mathbb{F}_{q}$-isogenous to the $g$-th power of a supersingular elliptic curve with all its endomorphisms defined over $\mathbb{F}_{q}$. All these assertions can be found in [Ta 66].

Given an $\mathbb{F}_{q}$-polarization $\lambda: A \rightarrow \widehat{A}$, we consider the Rosati involution, defined on $\operatorname{End}^{0}(A)$ by $\psi \mapsto \psi^{\prime}=\lambda^{-1} \circ \widehat{\psi} \circ \lambda$. It belongs to $\mathbb{Q}(\varphi)$. The Verschiebung, $\varphi^{\prime}$, is an element of $\operatorname{End}_{\mathbb{F}_{q}}(A)$ and satisfies $\varphi \circ \varphi^{\prime}=q$.

If $A$ is $\mathbb{F}_{q}$-simple, then $\mathbb{Q}(\varphi)$ is a number field and the Rosati involution agrees on $\mathbb{Q}(\varphi)$ with the complex conjugation $c$, for all embeddings of $\mathbb{Q}(\varphi)$ into $\overline{\mathbb{Q}}$. The class in the Brauer group of $\mathbb{Q}(\varphi)$ of the simple algebra $\operatorname{End}_{\mathbb{F}_{q}}^{0}(A)$ is characterized by the local invariants $i_{\wp}=f_{\wp} \operatorname{ord}_{\wp}(\varphi) / n$ at each prime $\wp$ over $p$ in $\mathbb{Q}(\varphi)$ (here, $f_{\wp}$ stands for the residual degree at $\wp$ ); on each real prime, the local invariant is equal to $1 / 2$; on the remaining primes, the algebra splits. The lowest common denominator $e$ of all the invariants $i_{\wp}$ is the period of the endomorphism algebra $\operatorname{End}_{\mathbb{F}_{q}}^{0}(A)$. The characteristic polynomial of $\varphi$ acting on the Tate module equals the $e$-th power of the $\mathbb{Q}$-irreducible polynomial of $\varphi$ (cf. [Ta 66], [Wa 69]).

We fix an algebraic closure $\overline{\mathbb{Q}}$ of $\mathbb{Q}$. An element $\alpha \in \overline{\mathbb{Q}}$ is called a Weil $q$-number if $|\alpha|=q^{1 / 2}$, for all archimedian absolute values $|\cdot|$ on $\overline{\mathbb{Q}}$. Each Weil $q$-number $\alpha$ determines, up to isogenies, an $\mathbb{F}_{q}$-simple abelian variety $A / \mathbb{F}_{q}$ such that the $\mathbb{Q}$-irreducible polynomial of $\varphi$ equals the $\mathbb{Q}$-irreducible polynomial of $\alpha$. This assignment establishes a one to one correspondence between the conjugation classes of Weil $q$-numbers and the $\mathbb{F}_{q}$-isogenies classes of $\mathbb{F}_{q}$-simple abelian varieties which are $\mathbb{F}_{q}$-defined (cf. [Ta 68]).

Let $\alpha_{1}, \alpha_{2}$ be two Weil $q$-numbers such that $\mathbb{Q}\left(\alpha_{1}\right)=\mathbb{Q}\left(\alpha_{2}\right)$. If the ideals $\left(\alpha_{1}\right),\left(\alpha_{2}\right)$ in the ring of integers of $K:=\mathbb{Q}\left(\alpha_{1}\right)$ coincide, then their associated abelian varieties are $\overline{\mathbb{F}}_{q}$-isogenous. If $\left(\alpha_{1}\right)=\left(\alpha_{2}\right)$, then there exists a unit $\varepsilon \in K$ such that $\alpha_{2}=\varepsilon \alpha_{1}$; since $|\varepsilon|=1$, we have that $\varepsilon$ must be a root of unity. If $\varepsilon^{s}=1$, then $\alpha_{1}^{s}=\alpha_{2}^{s}$. The characteristic polynomials of the relative Frobenius of both abelian varieties over $\mathbb{F}_{q^{s}}$ are equal. Thus, the varieties are $\mathbb{F}_{q^{s}}$-isogenous. We note that the abelian variety associated to a Weil $q$-number $\alpha$ is $\overline{\mathbb{F}}_{q}$-isogenous to a power of a supersingular elliptic curve if and only if the ideals $\left(\alpha^{2}\right)$ and (q) do coincide.

In the sequel the term isogenous will indicate $\overline{\mathbb{F}}_{q}$-isogenous. 


\section{Some relations between $\operatorname{End}^{0}(A)$ and $r(A)$}

In this section we give some propositions which relate the $p$-rank $r(A)$ to the structure of the $\mathbb{Q}$-algebra $\operatorname{End}_{\mathbb{F}_{q}}^{0}(A)$.

Let $P(X):=\operatorname{det}\left(\varphi-X\right.$ Id $\left.\mid V_{\ell}(A)\right)$, which is a polynomial with integer coefficients independent of $\ell$. If $\alpha_{i}, 1 \leq i \leq g$, denote its complex roots, then we have that $\left|\alpha_{i}\right|=q^{1 / 2}$ and $\prod_{i=1}^{2 g} \alpha_{i}=q^{g}$. The real roots of $P(X)$ have even multiplicity and we can order all the roots so that $\alpha_{i+g}=\bar{\alpha}_{i}=q / \alpha_{i}$, for $1 \leq i \leq g$. For such an order we write $\beta_{i}:=\alpha_{i}+q / \alpha_{i}$. The polynomial $Q(X):=\prod_{i=1}^{g}\left(X-\beta_{i}\right)$ has integer coefficients too. We have

$$
Q(X)^{2}=\operatorname{det}\left(\varphi+\varphi^{\prime}-X \operatorname{Id} \mid V_{\ell}(A)\right), \quad P(X)=X^{g} Q\left(X+\frac{q}{X}\right) .
$$

Then $P(X)(\bmod p)=X^{g} Q(X)(\bmod p)$. The following results are known (cf. [Ma 65], [St 79], [Ba-Go 97]).

\subsection{Proposition.}

i) $r(A)$ is the sum of the multiplicities of the non-zero roots of the $(\bmod p)$-reduced characteristic polynomial $P(X)$ and, hence, of those of the polynomial $Q(X)(\bmod p)$.

ii) We have $r(A)=\#\left\{\beta_{i} \notin \wp \mid 1 \leq i \leq g\right\}=\#\left\{\alpha_{i} \notin \wp \mid 1 \leq i \leq 2 g\right\}$, where $\wp$ is a prime ideal over $p$ in the ring of integers of $\mathbb{Q}\left(\left\{\alpha_{i}\right\}\right)$.

By using the results displayed in the proposition and in the previous section, we obtain

3.2. Proposition. Let $A / \mathbb{F}_{q}$ be an $\mathbb{F}_{q}$-simple abelian variety. Then:

i) A is ordinary if and only if the ideals $(\varphi),\left(\varphi^{\prime}\right)$ (equivalently, the ideals $\left.\left(\varphi+\varphi^{\prime}\right),(p)\right)$ are relatively prime in $\mathbb{Q}(\varphi)$.

ii) $r(A)=0$ if and only if every prime $\wp \mid(p)$ divides $(\varphi)$ in $\mathbb{Q}(\varphi)$ (equivalently, divides $\left(\varphi+\varphi^{\prime}\right)$ ).

iii) $A$ is isogenous to a power of a supersingular elliptic curve if and only if $(\varphi)=\left(\varphi^{\prime}\right)$.

iv) The period e of $\operatorname{End}_{\mathbb{F}_{q}}^{0}(A)$ in the Brauer group divides $r(A)$. 
From now on, we assume that $A / \mathbb{F}_{q}$ is $\mathbb{F}_{q}$-simple. As usual, we will say that $A$ is absolutely simple if it is $k$-simple.

3.3. Corollary. If there exists a prime ideal $\wp \mid(p)$ such that $\wp^{c}=\wp$, then $A$ is non ordinary. If $A$ is not isogenous to a power of a supersingular elliptic curve, then there exists a prime ideal $\wp \mid(p)$ such that $\wp \neq \wp^{c}$.

Proof: Since $\varphi^{c}=\varphi^{\prime}$, if $\wp=\wp^{c}$ we shall have $\wp \mid\left(\varphi+\varphi^{\prime}\right)$ and $A$ will be non ordinary. If every prime ideal over $p$ is invariant under complex conjugation, then $(\varphi)=\left(\varphi^{\prime}\right)$.

3.4. Proposition. Assume that $q=p$. Then, we have

i) If $A$ is non ordinary, there exists a prime $\wp \mid(p)$ in $\mathbb{Q}(\varphi)$ which ramifies.

ii) If $r(A)=0$, every prime $\wp \mid(p)$ in $\mathbb{Q}(\varphi)$ does ramify.

iii) $\operatorname{End}_{\mathbb{F}_{p}}^{0}(A)$ is a totally imaginary number field if and only if $A$ is not isogenous to the square of a supersingular elliptic curve.

Proof: If $A$ is non ordinary there exists a prime $\wp \mid(p)$ such that $\wp \mid(\varphi)$ and $\wp \mid\left(\varphi^{\prime}\right)$. Since $\varphi \varphi^{\prime}=p$, it follows that $\wp$ ramifies. If $r(A)=0$, the condition if fulfilled by all the primes which divide $(p)$. Let us prove iii). We recall that $A$ is $\mathbb{F}_{p}$-simple. Since $n=1$, the period $e$ in the Brauer group is 1 or 2 , depending on whether $\mathbb{Q}(\varphi)$ is totally imaginary or not. Thus, $e=1$ if and only if $\operatorname{End}_{\mathbb{F}_{p}}^{0}(A)$ is a totally imaginary number field. The value $e$ is equal to 2 if and only if $\varphi^{2}=p$. In this case, the characteristic polynomial of $\varphi$ is $\left(X^{2}-p\right)^{2}$, which has associated an $\mathbb{F}_{p^{-}}$-simple abelian variety $\mathbb{F}_{p^{2}}$-isogenous to the square of a supersingular elliptic curve defined over $\mathbb{F}_{p^{2}}$.

3.5. Proposition. If $r(A)$ is prime to $g$, then $\operatorname{End}_{\mathbb{F}_{q}}^{0}(A)$ is a commutative field.

Proof: If $\operatorname{dim} A=1$, the statement is known and, therefore, we may assume that $g>1$. The field $\mathbb{Q}(\varphi)$ is either totally imaginary, equal to $\mathbb{Q}(\sqrt{p})$, or equal to $\mathbb{Q}$. Since $A$ is $\mathbb{F}_{q}$-simple, the last possibility is excluded in our case. Thus $[\mathbb{Q}(\varphi): \mathbb{Q}]$ is even and $e$ divides $g$, because $e[\mathbb{Q}(\varphi): \mathbb{Q}]=2 g$. Since $e$ also divides $r(A)$, it follows that $e=1$.

The following theorem allows us to characterize, by means of the commutativity of $\operatorname{End}^{0}(A)$, the ordinary character of those absolutely simple 
abelian varieties whose $\mathbb{Q}$-algebra $\operatorname{End}^{0}(A)$ has as center an abelian extension of $\mathbb{Q}$. Note that, since the center of the endomorphism algebra of an elliptic curve is always abelian over $\mathbb{Q}$, the ordinary character of an elliptic curve, $E$, is equivalent to the commutativity of $\operatorname{End}^{0}(E)$.

\subsection{Theorem.}

i) If $A$ is ordinary, then $\operatorname{End}_{\mathbb{F}_{q}}^{0}(A)$ is commutative and, therefore, $\operatorname{End}_{\mathbb{F}_{q}}^{0}(A)=\mathbb{Q}(\varphi)$. In particular, if $A$ is ordinary and absolutely simple, $\operatorname{End}^{0}(A)$ is commutative.

ii) If $p$ splits completely in $\mathbb{Q}(\varphi)$ and $\operatorname{End}_{\mathbb{F}_{q}}^{0}(A)$ is commutative, then A is ordinary.

iii) If $A$ is absolutely simple and the center $K$ of $\operatorname{End}^{0}(A)$ is an abelian extension of $\mathbb{Q}$, then $p$ splits completely in $K$.

Proof: i) Let us assume that $A$ is ordinary. The ideals $(\varphi),\left(\varphi^{\prime}\right)$ are relatively prime in $\mathbb{Q}(\varphi)$ by 3.2 i) and $\varphi \varphi^{\prime}=p^{n}$. Thus, for all primes $\wp \mid(p)$ in $\mathbb{Q}(\varphi)$, we have that $\operatorname{ord}_{\wp} \varphi$ is zero or a positive multiple of $n$ and, so, $i_{\wp} \in \mathbb{Z}$. The field $\mathbb{Q}(\varphi)$ has no real primes, because if $\varphi$ were real, then $\varphi= \pm q^{1 / 2}$ and $A$ would be non ordinary. Since all the local invariants of $\operatorname{End}_{\mathbb{F}_{q}}^{0}(A)$ are trivial, its Brauer period must be $e=1$; i.e., $\operatorname{End}_{\mathbb{F}_{q}}^{0}(A)=\mathbb{Q}(\varphi)$. This line of reasoning parallels that used in $[\mathbf{Y u ~ 7 8}]$ for the case of the jacobian of a curve.

Let us now prove ii). If $\operatorname{End}_{\mathbb{F}_{q}}^{0}(A)$ is commutative and $p$ splits completely, then for all primes $\wp \mid(p)$ in $\mathbb{Q}(\varphi)$ we have $i_{\wp}=\operatorname{ord}_{\wp} \varphi / n \in \mathbb{Z}$ and $\operatorname{ord}_{\wp} \varphi$ is zero or $n$. Since $n=\operatorname{ord}_{\wp} \varphi+\operatorname{ord}_{\wp} \varphi^{\prime}$, we get that $(\varphi)$ and $\left(\varphi^{\prime}\right)$ are relatively prime and $A$ is ordinary.

Let us see iii). We may assume without loss of generality that $\operatorname{End}^{0}(A)$ is equal to $\operatorname{End}_{\mathbb{F}_{q}}^{0}(A)$. Then $K=\mathbb{Q}\left(\varphi^{s}\right)$, for all $s>0$. The $\mathbb{Q}$-irreducible polynomial of $\varphi$ is $\prod_{\sigma \in G}(X-\sigma(\varphi))$, where $G=\operatorname{Gal}(K / \mathbb{Q})$. Let $\wp \mid(p)$ be a prime in $K$ and let $\mathcal{D}$ denote its decomposition group in $K / \mathbb{Q}$. Assume that $p$ does not split completely in $K$. Then we can take $\sigma \in \mathcal{D} \backslash\{\operatorname{Id}\}$ and the ideals $(\sigma(\varphi))$ and $(\varphi)$ coincide, since $K / \mathbb{Q}$ is abelian. There exists a root of unity $\varepsilon \in K$ such that $\varphi=\varepsilon \sigma(\varphi)$. If $s>1$ is the order of $\varepsilon$, then $\varphi^{s}=\sigma\left(\varphi^{s}\right)$. Then $\left[\mathbb{Q}\left(\varphi^{s}\right): \mathbb{Q}\right]<[\mathbb{Q}(\varphi): \mathbb{Q}]$, which is a contradiction.

If $A / \mathbb{F}_{q}$ and $B / \mathbb{F}_{q}$ are absolutely simple abelian varieties of dimension $g>1$, then $A$ and $B$ can have $\mathbb{Q}$-isomorphic endomorphism algebras by means of a homomorphism $\Phi: \operatorname{End}^{0}(A) \stackrel{\sim}{\rightarrow} \operatorname{End}^{0}(B)$ such that $\Phi\left(\varphi_{A}\right)$ is none of the conjugates of $\varphi_{B}$. If this is the case, $A$ and $B$ are not $\mathbb{F}_{q}$-isogenous. Nevertheless, as the following theorem shows, they have the same $p$-rank when $g=2$. 
3.7. Theorem. Let $A / \mathbb{F}_{q}$ be an absolutely simple abelian variety of dimension $g<3$. We have

i) If $g=1$, then $\operatorname{End}^{0}(A)$ determines $A$ up to isogenies.

ii) If $g=2$, then $\operatorname{End}^{0}(A)$ is a commutative field which determines $r(A)$. If, moreover, $p$ does not split completely in $\operatorname{End}^{0}(A)$, then $\operatorname{End}^{0}(A)$ determines $A$ up to isogenies.

Proof: The assertion i) is well known. We assume, without loss of generality, that $\operatorname{End}^{0}(A)=\operatorname{End}_{\mathbb{F}_{q}}^{0}(A)$. Thus, $\mathbb{Q}(\varphi)$ is the center of $\operatorname{End}^{0}(A)$.

For all abelian varieties $A / \mathbb{F}_{q}$ of dimension 2 the condition $r(A)=0$ is equivalent to the fact that $A$ is isogenous to the square of a supersingular elliptic curve. Thus, if $A$ is absolutely simple, either $A$ is ordinary or $r(A)=1$.

Assume that $\operatorname{dim} A=2$ and that $A$ is absolutely simple. Since, in particular, $A$ is not isogenous to a power of a supersingular elliptic curve, the field $K:=\mathbb{Q}(\varphi)$ is totally imaginary and the ideals $(\varphi),\left(\varphi^{\prime}\right)$ are different, by $3.2 \mathrm{iii)}$.

In order to show that $\operatorname{End}^{0}(A)$ is a commutative field, we prove that $e=1$. Since $e[K: \mathbb{Q}]=4$ and $K \neq \mathbb{Q}, e=2$ or $e=1$. Assume that $e=2$. Then $[K: \mathbb{Q}]=2$ and the prime $p$ splits completely in $K$ by 3.6 iii). We have that $(p)=\wp \wp^{c}$. The ideal $(\varphi)$ is $\wp^{i}\left(\wp^{c}\right)^{n-i}$, for some $i$ such that $0 \leq i \leq n$, and the corresponding local invariants are $i / n$, $(n-i) / n$. Since $e=2$, we have that $i=n / 2$ and $(\varphi)=\left(\varphi^{\prime}\right)$, which leads to a contradiction. Thus, $e=1$ and $[K: \mathbb{Q}]=4$.

Let $L:=\mathbb{Q}\left(\varphi+\varphi^{\prime}\right)$, which is a quadratic extension of $\mathbb{Q}$. By 3.3 , there exists a prime ideal $\wp_{1} \mid(p)$ in $K$ such that $\wp_{1} \neq \wp_{1}^{c}$. This yields the following possibilities for the splitting type of $(p)$ in $K$ :

a) $(p)=\wp_{1}^{2}\left(\wp_{1}^{c}\right)^{2} \quad(p$ ramifies in $L)$,

b) $(p)=\wp_{1} \wp_{1}^{c} \quad(p$ is inert in $L)$,

c) $(p)=\wp_{1} \wp_{1}^{c} \wp_{2}^{s}, 1 \leq s \leq 2,(p$ splits completely in $L$ and not in $K)$,

d) $(p)=\wp_{1} \wp_{1}^{c} \wp_{2} \wp_{2}^{c} \quad(p$ splits completely in $K)$.

In case a), the ideal $(\varphi)$ is $\wp^{i}\left(\wp^{c}\right)^{2 n-i}, 0 \leq i \leq 2 n$. The local invariants $i / n,(2 n-i) / n$ are integers if and only if $i \in\{0, n, 2 n\}$. The case $i=n$ is not possible, since $(\varphi),\left(\varphi^{\prime}\right)$ would coincide. Thus, $(\varphi)$ is equal to $\wp^{2 n}$ or $\left(\wp^{c}\right)^{2 n}$. The two possible ideals are conjugated and, therefore, they correspond to isogenous abelian varieties. Thus, the $p$-rank of $A$ is determined. In this particular case, $A$ is ordinary, since $(\varphi)$ and $\left(\varphi^{\prime}\right)$ are relatively prime in $K$ and we apply $3.2 \mathrm{i}$ ). 
In case b), we have that $(\varphi)$ is $\wp_{1}^{n}$ or $\left(\wp_{1}^{c}\right)^{n}$. The two ideals are conjugated and they correspond to isogenous abelian varieties, which are ordinary.

In case c), we have that $(\varphi)$ is $\wp_{1}^{n} \wp_{2}^{s n / 2}$ or $\left(\wp_{1}^{c}\right)^{n} \wp_{2}^{s n / 2}$. The two solutions are conjugated and they correspond to isogenous abelian varieties, which are not ordinary because $\wp_{2}=\wp_{2}^{c}$. Thus $r(A)=1$.

In case d), the ideal $(\varphi)$ is equal to $\wp_{1}^{n} \wp_{2}^{n}, \wp_{1}^{n}\left(\wp_{2}^{c}\right)^{n},\left(\wp_{1}^{c}\right)^{n} \wp_{2}^{n}$ or $\left(\wp_{1}^{c}\right)^{n}\left(\wp_{2}^{c}\right)^{n}$. These four solutions correspond to two possible ordinary abelian varieties which are not isogenous.

We see that in all cases $r(A)$ is determined by $\operatorname{End}^{0}(A)$. If $p$ does not split completely in $\operatorname{End}^{0}(A)$ then only cases a), b) or c) are possible. In all of them, $A$ is determined up to isogenies by $\operatorname{End}^{0}(A)$. We remark that the first claim of ii) can be deduced from [Oo 87, 6.5].

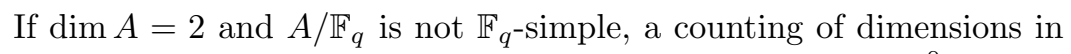
each possible splitting type of $A$ shows that the $\mathbb{Q}$-algebra $\operatorname{End}^{0}(A)$ also determines $r(A)$.

\section{An example}

In this section we will give an example of two absolutely simple abelian varieties of dimension 3 which have isomorphic endomorphism algebras but different $p$-ranks.

Let $\alpha$ be a Weil $q$-number. For each positive integer $m$, we denote by $A_{m}$ an abelian variety associated to the Weil $q^{m}$-number $\alpha^{m}$. Let $e_{m}$ be the Brauer period of $\operatorname{End}_{\mathbb{F}_{q} m}^{0}\left(A_{m}\right)$. We have the following equivalent conditions:

i) $A_{1} / \mathbb{F}_{q}$ is absolutely simple.

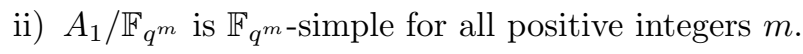

iii) $\operatorname{dim} A_{1}=\operatorname{dim} A_{m}$ for all positive integers $m$.

iv) $[\mathbb{Q}(\alpha): \mathbb{Q}] e_{1}=\left[\mathbb{Q}\left(\alpha^{m}\right): \mathbb{Q}\right] e_{m}$ for all positive integers $m$.

Since $\mathbb{Q}(\alpha) / \mathbb{Q}$ is a finite extension, there exists a positive integer $t$ such that $\mathbb{Q}\left(\alpha^{t}\right)=\mathbb{Q}\left(\alpha^{t m}\right)$ for all positive integers $m$. For this $t$, we have that $e_{t}=e_{t m}$ for all $m$ and, thus, $A_{t}$ is absolutely simple. The abelian variety $A_{1}$ is absolutely simple if and only if $\operatorname{dim} A_{1}=\operatorname{dim} A_{t}=$ $\left[\mathbb{Q}\left(\alpha^{t}\right): \mathbb{Q}\right] e_{t} / 2$ and, in this case, we have that $\operatorname{End}^{0}\left(A_{1}\right)=\operatorname{End}_{\mathbb{F}_{q^{t}}}^{0}\left(A_{t}\right)$. In particular, if $\mathbb{Q}\left(\alpha^{m}\right)=\mathbb{Q}(\alpha)$ for all $m$, then $A_{1}$ is absolutely simple and $\operatorname{End}^{0}\left(A_{1}\right)=\operatorname{End}_{\mathbb{F}_{q}}^{0}\left(A_{1}\right)$. This is the condition which we will use in our example. 
The next proposition yields a criterion which makes it easy to determine whether an abelian variety $A / \mathbb{F}_{q}$, associated to a Weil $q$-number $\alpha$ such that $[\mathbb{Q}(\alpha): \mathbb{Q}]=6$, is absolutely simple.

4.1. Proposition. Let $\alpha$ be a Weil $q$-number such that $[\mathbb{Q}(\alpha): \mathbb{Q}]=6$. If there exists a positive integer s such that $\mathbb{Q}\left(\alpha^{s}\right) \subsetneq \mathbb{Q}(\alpha)$, then the $\mathbb{Q}$-irreducible polynomial of $\alpha, P(x)$, is of type $X^{6}+a X^{3}+q^{3}$ or $\mathbb{Q}(\alpha)=\mathbb{Q}\left(\mu_{7}\right)$. If the polynomial $P(x)$ is of type $X^{6}+a X^{3}+q^{3}$, then $\mathbb{Q}\left(\alpha^{3}\right) \subsetneq \mathbb{Q}(\alpha)$.

Proof: For each positive integer $s$, the field $\mathbb{Q}\left(\alpha^{s}\right)$ is real if and only if $\alpha^{s}= \pm q^{1 / 2}$. In this case $\left[\mathbb{Q}\left(\alpha^{s}\right): \mathbb{Q}\right]$ is 2 or 1 ; otherwise, $\mathbb{Q}\left(\alpha^{s}\right)$ is totally imaginary. We write $K:=\mathbb{Q}(\alpha)$ and we denote by $L:=\mathbb{Q}(\alpha+\bar{\alpha})$ the largest real subfield of $K$. The field $L$ is the only subfield of $K$ which has dimension 3 over $\mathbb{Q}$ and, thus, $\left[\mathbb{Q}\left(\alpha^{s}\right): \mathbb{Q}\right] \neq 3$ for all positive integers $s$.

Let $m$ be the smallest positive integer such that $\mathbb{Q}\left(\alpha^{m}\right) \subsetneq K$. The integer $m$ is odd, otherwise $\left[\mathbb{Q}\left(\alpha^{m / 2}\right): \mathbb{Q}\right]=6$ and, then $\left[\mathbb{Q}\left(\alpha^{m}\right): \mathbb{Q}\right]=3$. We consider two cases.

1) The Weil $q$-number $\alpha$ is equal to $q^{1 / 2} \zeta$, where $\zeta$ is a root of unity.

We assume that $q^{1 / 2} \in \mathbb{Z}$. Then $K=\mathbb{Q}(\zeta)$ and $[\mathbb{Q}(\zeta): \mathbb{Q}]=6$. Therefore, $K=\mathbb{Q}\left(\mu_{7}\right)$ or $K=\mathbb{Q}\left(\mu_{9}\right)$. If $K=\mathbb{Q}\left(\mu_{9}\right)$, then the polynomial $P(X)$ is equal to $X^{6} \pm q^{3 / 2} X^{3}+q^{3}$.

If $q^{1 / 2} \notin \mathbb{Z}$ then $\alpha^{2}=q \zeta^{2}$. We have that $\mathbb{Q}(\alpha)=\mathbb{Q}\left(\alpha^{2}\right)$, because $m>2$, and $K$ is equal to $\mathbb{Q}\left(\mu_{7}\right)$ or $\mathbb{Q}\left(\mu_{9}\right)$. Since $m$ is odd, we have that $q^{m / 2} \notin \mathbb{Q}$ and $\left[\mathbb{Q}\left(\alpha^{m}\right): \mathbb{Q}\right]=2$. The field $\mathbb{Q}\left(\mu_{9}\right)$ only contains the quadratic field $\mathbb{Q}(\sqrt{-3})$. Thus, if $K=\mathbb{Q}\left(\mu_{9}\right)$ then $p=3$ and $\alpha= \pm(-q)^{1 / 2} \zeta_{1}$, where $\zeta_{1}$ is a primitive 9 -th root of unity; in this case, the polynomial $P(x)$ is equal to $X^{6} \pm\left(3 q^{3}\right)^{1 / 2} X^{3}+q^{3}$.

2) $\mathbb{Q}\left(\alpha^{s}\right) \neq \mathbb{Q}$ for all positive integers $s$.

In this case $\mathbb{Q}\left(\alpha^{m}\right) / \mathbb{Q}$ is an imaginary quadratic extension and there exist two primitive $m$-th roots of unity, $\zeta_{1}$ and $\zeta_{2}$, such that

$$
P(X)=(X-\alpha)\left(X-\alpha \zeta_{1}\right)\left(X-\alpha \zeta_{2}\right)(X-\bar{\alpha})\left(X-\bar{\alpha} \zeta_{1}^{-1}\right)\left(X-\bar{\alpha} \zeta_{2}^{-1}\right)
$$

We denote by $\widetilde{K}$ the normal closure of $K$. We write $G:=\operatorname{Gal}(\widetilde{K} / \mathbb{Q})$, $H:=\left\{\sigma \in G \mid \sigma\left(\alpha \zeta_{2}\right)=\alpha \zeta_{2}\right\}$. We note that if $\sigma\left(\alpha \zeta_{2}\right)=\alpha \zeta_{2}$ and $\sigma\left(\alpha \zeta_{1}\right)=\alpha \zeta_{1}$, then $\sigma=$ Id since the complex conjugation is in the center of $G$. We consider the following possibilities: 
i) $H \neq\{\operatorname{Id}\}$. In this case, there exists $\sigma \in G$ such that

$$
\sigma\left(\alpha \zeta_{2}\right)=\alpha \zeta_{2}, \quad \sigma\left(\alpha \zeta_{1}\right)=\alpha, \quad \sigma(\alpha)=\alpha \zeta_{1} .
$$

Therefore, $\sigma(\alpha)=\alpha \zeta_{1}$ and $\sigma^{2}(\alpha)=\alpha$. Since $\left\{\sigma \in G \mid \sigma\left(\alpha \zeta_{1}\right)=\right.$ $\left.\alpha \zeta_{1}\right\} \neq\{\operatorname{Id}\}$, there exists $\tau \in G$ such that $\tau(\alpha)=\alpha \zeta_{2}$ and $\tau^{2}(\alpha)=\alpha$. The conditions $\sigma^{2}(\alpha)=\tau^{2}(\alpha)=\alpha$ imply that $\sigma, \tau$ coincide in $\mathbb{Q}\left(\mu_{m}\right)$ with the complex conjugation. Thus $(\sigma \circ \tau)(\alpha)=\alpha \zeta_{1} \zeta_{2}^{-1}$. Since $\zeta_{1} \zeta_{2}^{-1} \in\left\{1, \zeta_{1}, \zeta_{2}\right\}$ and $\zeta_{1} \notin\left\{1, \zeta_{2}\right\}$, we have that $\zeta_{1}=\zeta_{2}^{2}$. Using $\tau \circ \sigma$, we obtain that $\zeta_{2}=\zeta_{1}^{2}$. Thus, $\zeta_{1}, \zeta_{2} \in \mu_{3}$ and $m=3$.

ii) $H=\{\operatorname{Id}\}$. In this case, $\widetilde{K}=K$. The field $\mathbb{Q}\left(\mu_{m}\right)$ is totally imaginary because $m$ is odd, and thus, $\left[\mathbb{Q}\left(\mu_{m}\right): \mathbb{Q}\right]$ can only be equal to 6 or 2 . Therefore, $K=\mathbb{Q}\left(\mu_{7}\right)$ or $m=3$.

If $m=3$ then $P(X)=\left(X^{3}-\alpha^{3}\right)\left(X^{3}-\bar{\alpha}^{3}\right)=X^{6}+a X^{3}+q^{3}$. It is clear that if $P(X)=X^{6}+a X^{3}+q^{3}$ then $\mathbb{Q}\left(\alpha^{3}\right) \subsetneq \mathbb{Q}(\alpha)$.

4.2. Example. We consider the modular curves $X_{0}(41) / \mathbb{Q}$, $X_{0}(41) / \mathbb{F}_{3}$, which have genus 3 . Let $A$ denote the jacobian of $X_{0}(41) / \mathbb{F}_{3}$.

From the tables of Wada, we see that the characteristic polynomial of the Hecke operator $T_{3}$ acting in $S_{2}\left(X_{0}(41)\right)$ is $Q(X)=X^{3}-4 X+2$, which is $\mathbb{Q}$-irreducible. We consider the natural action of $T_{3}$ as endomorphism of $J_{0}(41)$, the jacobian of $X_{0}(41)$, and its $(\bmod 3)$-reduction, $\widetilde{T}_{3}$, as endomorphism of $A$. The $\mathbb{Q}$-irreducible polynomial of $\widetilde{T}_{3}$ acting in $\Omega_{1}(A)$ is $Q(X)$.

The real field $L=\mathbb{Q}\left(\widetilde{T}_{3}\right)$ has discriminant $2^{2} \cdot 37$ and, thus, $L \not \subset \mathbb{Q}\left(\mu_{7}\right)$. The congruence of Eichler-Shimura establishes that $\widetilde{T}_{3}=\varphi+\varphi^{\prime}$. Then $\mathbb{Q}(\varphi) / L$ is an imaginary quadratic extension and the $\mathbb{Q}$-irreducible polynomial of $\varphi$ is

$$
P(X)=X^{3} Q(X+3 / X)=X^{6}+5 X^{4}+2 X^{3}+15 X^{2}+27 .
$$

By $3.1 \mathrm{ii}), r(A)=3$, because the $(\bmod 3)$-reduced polynomial $Q(x)$ has three non zero roots. Since $A$ is defined over $\mathbb{F}_{3}$, then $e=1$ and $\operatorname{End}_{\mathbb{F}_{3}}^{0}(A)=\mathbb{Q}(\varphi)$.

Let $\alpha:=3 \varphi$, which is a Weil $3^{3}$-number. We have that $\mathbb{Q}\left(\alpha^{m}\right)=$ $\mathbb{Q}\left(\varphi^{m}\right)$ for all positive integers $m$. Let $B / \mathbb{F}_{27}$ be the abelian variety associated to $\alpha$. It has $r(B)=0$, by $3.2 \mathrm{ii}$ ). The prime 3 is inert in $L$ and does not ramify in $\mathbb{Q}(\alpha)$. The ideal (3) is not prime in $\mathbb{Q}(\alpha)$ because $r(A) \neq 0$. Then, we have that $(3)=\wp \wp^{c}$ with $f_{\wp}=f_{\wp c}=3$ in $\mathbb{Q}(\alpha)$. Thus, the Brauer periode $e$ of $\operatorname{End}_{\mathbb{F}_{27}}^{0}(B)$ is 1. Therefore, $\operatorname{dim} B=3$ and $\operatorname{End}_{\mathbb{F}_{27}}^{0}(B)=\mathbb{Q}(\alpha)$. 
Since $P(X)$ is not of type $X^{6}+a X^{3}+3^{3}$ and $\mathbb{Q}(\varphi) \neq \mathbb{Q}\left(\mu_{7}\right)$, we have by 4.1 that $\mathbb{Q}(\varphi)=\mathbb{Q}\left(\varphi^{m}\right)$, for all positive integers $m$. Thus, $A$ and $B$ are absolutely simple and $\operatorname{End}^{0}(A), \operatorname{End}^{0}(B)$ are isomorphic to $\mathbb{Q}(\varphi)$.

\section{References}

[Ba-Go 97] P. Bayer And J. GonzÁlez, On the Hasse-Witt invariants of modular curves, Experiment. Math. 6 (1997), 57-76.

[De 41] M. Deuring, Die Typen der Multiplikatorenringe elliptischer Funktionenkörper, Abh. Math. Sem. Univ. Hamburg 14 (1941), 197-272.

[Go 97] J. GonzÁLEz, Hasse-Witt matrices for the Fermat curves of prime degree, Tôhoku Math. J. 49 (1997), 149-163.

[Ha 34] H. HAsse, Existenz separabler zyklischer unverzweigter Erweiterungskörpern vom Primzahlgrade $p$ über elliptischen Funktionenkörpern der Charakteristik $p$, J. Reine Angew. Math. 172 (1934), $77-85$.

[Ha-Wi36] H. Hasse AND E. Witt, Zyklische unverzweigte Erweiterungskörpern vom Primzahlgrade $p$ über einem algebraischen Funktionenkörpern der Charakteristik p, Monatsh. Math. Phys. 43 (1936), 477-492.

[Ma 65] J. I. Manin, The Hasse-Witt matrix of an algebraic curve, Amer. Math. Soc. Transl. Ser. 45 (1965), 245-264.

[Oo 87] F. OoRT, Endomorphism Algebras of Abelian Varieties, Algebraic Geometry and Commutative Algebra, in honour of M. Nagata 2 (1987), 469-502.

[Se 58] J. P. Serre, Sur la topologie des variétés algébriques en caractéristique $p$, Symp. Int. Top. Alg., México, p. 24-53, in "Euvres," vol. I, Springer.

[St 79] H. Stichtenoth, Die Hasse-Witt-Invariante eines Kongruenzfunktionenkörpers, Arch. Math. 33 (1979), 357-360.

[Ta 66] J. TATE, Endomorphisms of abelian varieties over finite fields, Invent. Math. 2 (1966), 134-144.

[Ta 68] J. TATE, Classes d'isogénie des variétés abéliennes sur un corps fini (d'après T. Honda), Sém. Bourbaki (1968/69), 95-110.

[Wa 69] W. C. Waterhouse, Abelian varieties over finite fields, Ann. Sci. École Norm. Sup. 2 (1969), 521-560. 
[Yu 78] N. YUI, On the Jacobian varieties of hyperelliptic curves, $J$. Algebra 52 (1978), 378-410.

Departament de Matemàtica Aplicada i Telemàtica Escola Universitària Politècnica de Vilanova i la Geltrú

Av. Victor Balaguer s/n

Vilanova i la Geltrú 08800

SPAIN

e-mail: josepg@mat.upc.es

Primera versió rebuda el 21 de gener de 1997, darrera versió rebuda el 3 d'abril de 1997 